\title{
First results of a uniform regional treatment protocol and registration for acute prosthetic join infection in the South-East of the Netherlands
}

\author{
Maud C. Kamp ${ }^{1,2}$, Robin W.T.M. van Kempen ${ }^{1}$, Loes Janssen ${ }^{3}$, M.C. (Marieke) van der Steen ${ }^{1,4}{ }^{凶}$, on behalf \\ of the Regional Prosthetic Joint Infection working group \\ 1. Department of Orthopaedic Surgery, Catharina Hospital Eindhoven, Postbus 1350, 5602 ZA Eindhoven, The Netherlands. \\ 2. Department of Orthopedic Surgery, Radboudumc, Postbus 9101, 6500 HB Nijmegen, the Netherlands. \\ 3. Department of Orthopedic Surgery, VieCuri Medical Centre, Postbus 1926, 5900 BX Venlo, The Netherlands \\ 4. Orthopaedic Center Máxima, Máxima Medical Center, Postbus 90052, 5600 PD Eindhoven, The Netherlands. \\ $\triangle$ Corresponding author: marieke.vd.steen@catharinaziekenhuis.nl. \\ (C) The authors. This is an open access article distributed under the terms of the Creative Commons Attribution (CC BY) license (https://creativecommons.org/licenses/ \\ by/4.0/). See http://ivyspring.com/terms for full terms and conditions.
}

Received: 2019.01.11; Accepted: 2019.04.30; Published: 2019.05.21

\begin{abstract}
Introduction: Prosthetic joint infection (PJI) is a relatively uncommon (average incidence $0.5-2 \%$ ) but devastating complication, with significant morbidity and leading to tremendously increased health care costs. In 2013, delegates from nine hospitals covering a large region in the South-East Netherlands composed one combined treatment protocol for acute PJI of total hip and knee arthroplasty (THA and TKA). This protocol was based on the definition of acute PJI according to Workgroup of the American Musculoskeletal Infection Society (MSIS) and the principles of debridement, antibiotics, irrigation and retention (DAIR).

Methods: Patients with a THA or TKA treated with DAIR because of suspicion of PJI were selected from the online PJI database. PJI was defined as at least two phenotypically identical pathogens, isolated in cultures from at least two separate tissues, obtained from the affected prosthetic joint. Acute PJls, occurring within 90 days after primary implantation, between January 2014 and December 2016, were analyzed. We analyzed the PJI incidence rate, patient clinical and microbiological characteristics of PJI, outcome of the DAIR treatment and adherence to the regional protocol.

Results: A total of 310 primary THA or TKA with a suspected PJI were registered in the regional PJI database, 236 met the definition of acute PJI, representing overall incidence of $1.12 \%$. Following the regional treatment protocol replacement of exchangeable parts took place in $45 \%$ in 2014 to $70 \%$ in 2016 . After 12 months follow-up, prosthesis retention was achieved in $87 \%$ and $3 \%$ of the patients died within one year after the primary surgery.

Conclusion: Results of the regional cohort are in line with the available literature. Regional collaboration and regular feedback on registered data resulted in better adherence to the combined treatment protocol. Despite our attempts to improve PJI care, PJI remains a serious complication of THA and TKA with a significant mortality rate and burden for the patient.
\end{abstract}

Key words: acute prosthetic join infection, debridement, antibiotics, irrigation, retention

\section{Introduction}

Prosthetic joint infection (PJI) is a relatively uncommon but devastating complication following total joint arthroplasty. The average reported incidence of PJI is $0.5-2 \%$. [1-3] PJI has a large negative impact on the patient and it causes significant morbidity, mortality, and leads to 
tremendously increased health care costs. [1,4-6] The Workgroup of the American Musculoskeletal Infection Society (MSIS) (2011) classified PJI in 3 groups, based on duration of symptoms and time after surgery. Acute infections manifest within 3 months after primary arthroplasty. Delayed (low-grade) infections usually become manifest 3-14 months after primary arthroplasty. Late (hematogenous) infections become manifest more than 2 years after primary arthroplasty. [1,2,7] Nowadays, debridement, antibiotics, irrigation and retention (DAIR) is the most widely performed treatment for acute PJI. [3,4,6] Success after DAIR treatment can be defined as eradication of infection with retention of the prosthesis and no occurrence of PJI-related mortality. A wide range of success rates (26-95\%) associated with patients who undergo debridement with implant retention has been reported in the literature. [3,4,6,8,9] Staphylococcus aureus (12-23\%) and coagulase-negative staphylococci $(30-43 \%)$ are the most commonly cultured microorganisms associated with acute PJI, followed by mixed flora $(10-11 \%)$, streptococci $(9-10 \%)$, gram-negative bacilli (3-6\%), enterococci $(3-7 \%)$, and anaerobes $(2-4 \%)$. $[1,3,10]$ Several attempts have been made to create treatment protocols for acute PJI. [e.g. $1,2,11,12]$ However, a recent survey showed that in approximately $25-30 \%$ of the Dutch Hospitals there is no protocol available for the treatment of cases suspected of acute PJI. [13] Furthermore, a lot of variety exists in the available protocols. $[13,14]$ As national (Dutch) registries are not developed for the registration of PJI but rather focus on implant survival, not all DAIR procedures -even if exchange of modular components took place- are reported in these registries. [13,15] This contributes to the underestimation of the rate of implant-related infections $[16,17]$.

In 2013, delegates from nine hospitals in the South-East Netherlands composed one combined diagnostic and treatment protocol for acute PJI based on the definition of acute PJI, according to MSIS and the principles of DAIR. With the aim to diminish unwanted variation and improve the quality of care around acute PJI of hip and knee arthroplasty. In order to evaluate the adherence to the treatment protocol and treatment outcome, the participating centers registered relevant aspects of treatment and outcome in a specially developed online registration system.

The purpose of this study was to give an overview of the first findings of this regional collaboration. We focus on PJI incidence rate, patient, clinical and microbiological characteristics of PJI, and outcome of the DAIR treatment. Furthermore, we evaluate the adherence to the regional protocol and describe our initiatives on how to improve this adherence.

\section{Methods}

\section{Regional treatment protocol and infection database}

In 2013, delegates from nine orthopedic departments of hospitals in the South-East Netherlands composed one combined diagnostic and treatment protocol for acute PJI. Figure 1. The applied definition of PJI follows the MSIS criteria for PJI. $[2,4,11,18]$ A PJI is present in case of a sinus tract communicating with the prosthesis. Or if a pathogen is isolated by culture from at least two separate tissue or fluid samples obtained from the affected prosthetic joint. Or if at least four of the minor criteria are present. Minor criteria are elevated serum erythrocyte sedimentation rate (ESR) and serum C-reactive protein (CRP) concentration; elevated synovial leukocyte count; elevated synovial polymorphonuclear neutrophil percentage (PMN\%); presence of purulence in the affected joint; isolation of microorganism in one culture or periprosthetic tissue or fluid; and more than five neutrophils per high-power field at $x 400$ magnification. [2,11,18] Within the regional treatment protocol early infections are defined as presentation of PJI within 3 months after placement of the prosthesis or as a result of a hematogenous seeding.

According to the regional treatment protocol all (acute) PJIs were treated following the regimen of DAIR. The procedure consists of incising the old scar or wound, obtaining tissue samples for multiple cultures (at least 6) from synovium, capsule, and interface. This is followed by the removal of old stiches and exchangeable components (e.g. polyethylene insert) of the prosthesis. Meticulous debridement and thorough irrigation by means of pulse lavage with at least $6 \mathrm{~L} \mathrm{NaCL}$ is performed before exchangeable components are replaced. $[1,3,6]$ In case of a high purulent infection at first debridement leaving behind gentamicin beads may be considered. Debridement and irrigation may be performed twice in the attempt to eradicate the PJI. Postoperatively, antibiotic treatment is based on bacterial susceptibility and determined in consultation with the medical microbiologist [8].

Patients with a total hip arthroplasty (THA), unipolar hip hemiarthroplasty, total knee arthroplasty (TKA) or revision THA/TKA, treated following DAIR are included in the regional PJI database. Data are acquired from the patients' electronic medical records and prospectively collected in the online regional PJI 
database. The data registered by each hospital in the online database include: specific operation information (e.g. type of prosthesis, operation side, operation technique), patient characteristics (e.g. sex, age, body mass index), risk factors (e.g. diabetes mellitus, malignancy, rheumatoid arthritis, smoking), clinical features (e.g. erythema, persistent wound leakage / dehiscence), date of debridement, cultured microorganisms and outcome after 3 and 12 months.

Each participating hospital has one or two contact persons (researcher / orthopedic surgeon) who is responsible for data registration, who can be contacted in case of questions by fellow surgeons and who participates in regular meetings with the other delegates. During these meetings uncertainties within the treatment protocol, challenging PJI cases and results of the registered data are discussed.

\section{Study design}

An analysis of prospectively registered data on patients diagnosed with an acute THA or TKA PJI, between 2014 and 2016, in nine hospitals in the South-East of the Netherlands was performed. Figure 1. The nine hospitals comprises a variety of academic, peripheral and private clinics. Data entry was reviewed (M.K., M.vd.S.) and controversies or contradictions were double-checked and if necessary corrected by the contact person at each hospital.

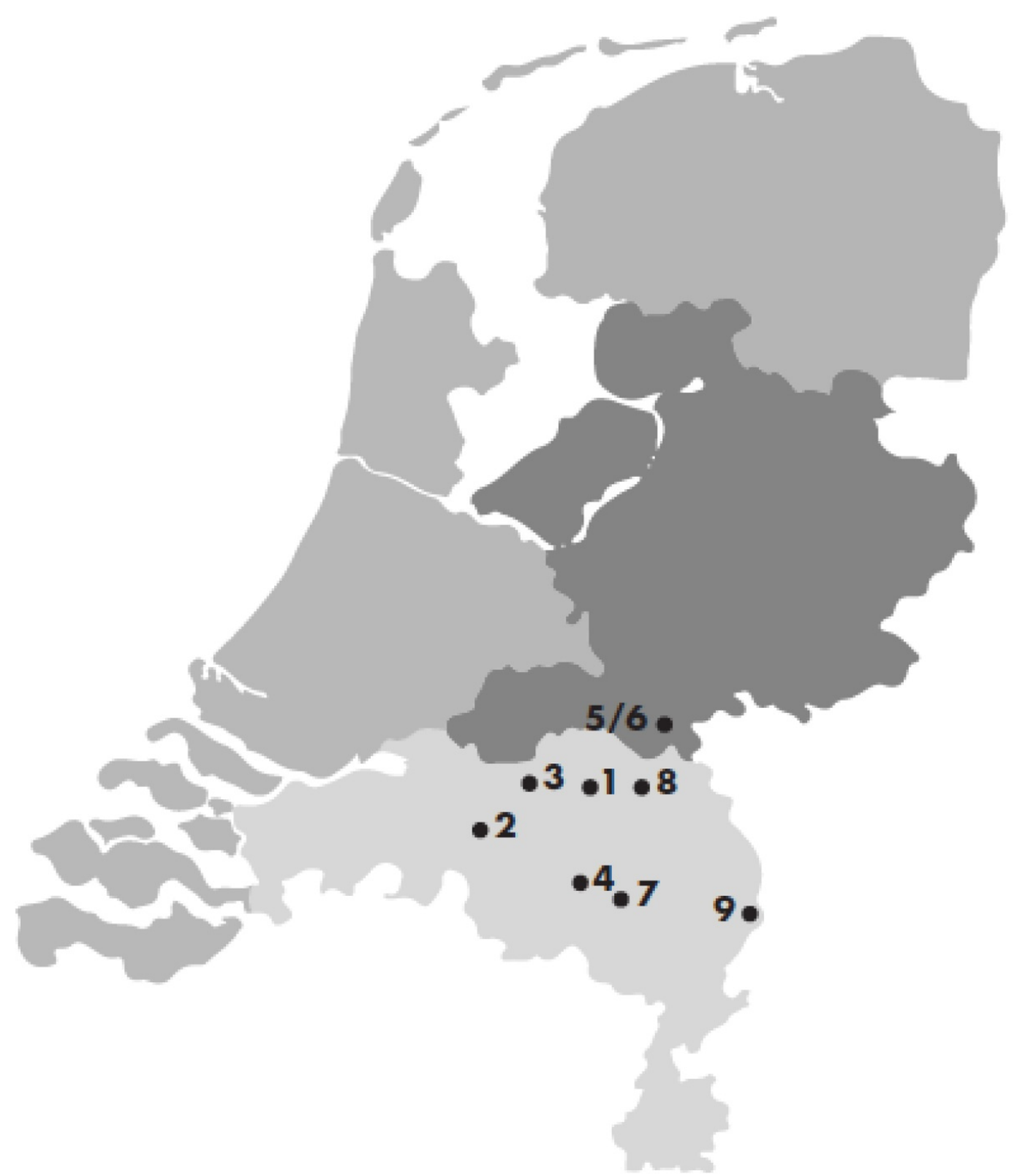

Figure 1. Regional infection cohort departments South-East Netherlands. 


\section{Participants}

For the current analyses we included acute PJI, classified as appearance of the first signs and symptoms of infection within 90 days after primary implantation between January 2014 and December 2016. [1,2] In addition, we excluded cases of revision arthroplasty, unipolar hip hemiarthroplasty, delayed or late infection (first signs and symptoms of infection $>90$ days after implantation) and cases were less than two perioperative tissue cultures were obtained to analyze.

\section{Outcome measures}

Patient characteristics, type of surgery and microbiology cultures were evaluated. Furthermore, we looked at mortality and treatment success. Treatment failure was defined as any further surgical procedure e.g. one/two-stage revision or Girdlestone situation at a follow-up of one year. Adherence to the protocol was investigated by analyzing the following key components; exchange of modular components (yes), number of cultures taken $(\geq 6)$ and number of DAIR treatments performed $(\leq 2)$.

\section{Statistical analysis}

The incidence was defined as the number of prosthetic joint infections between January 2014 and December 2016 in the South-East Netherlands per 100 primary total arthroplasty procedures. This was calculated as the ratio between the number of prosthetic joint infections from January 2014 to December 2016 and the number of primary total arthroplasties in the same period. Descriptive statistics were used to describe data in terms of incidence, totals and outcome. All continuous variables are reported as means and standard deviations, categorical data as totals and percentage.

Difference over the years in adherence to the protocol and outcome were tested by means of $x 2$ tests. Statistical analyses were performed using SPSS (version 21.0 RES Workspace Manager statistical software).

\section{Results}

\section{General}

Based on data from the Dutch Arthroplasty Register, 23.353 (approximately 14\% of all primary hip or knee arthroplasty in the Netherlands) [19] prosthetic hip or knee replacement procedures were performed between January 2014 and December 2016 in the nine participating hospitals. In the regional database a total of 426 suspicions of prosthetic joint infection were registered. We excluded 116 cases because of revision arthroplasty or unipolar prosthesis, leaving 310 primary TKA and THA with a suspected PJI. A total of 236 cases met the definition of acute PJI, representing an overall incidence of $1.12 \%$. Figure 2.
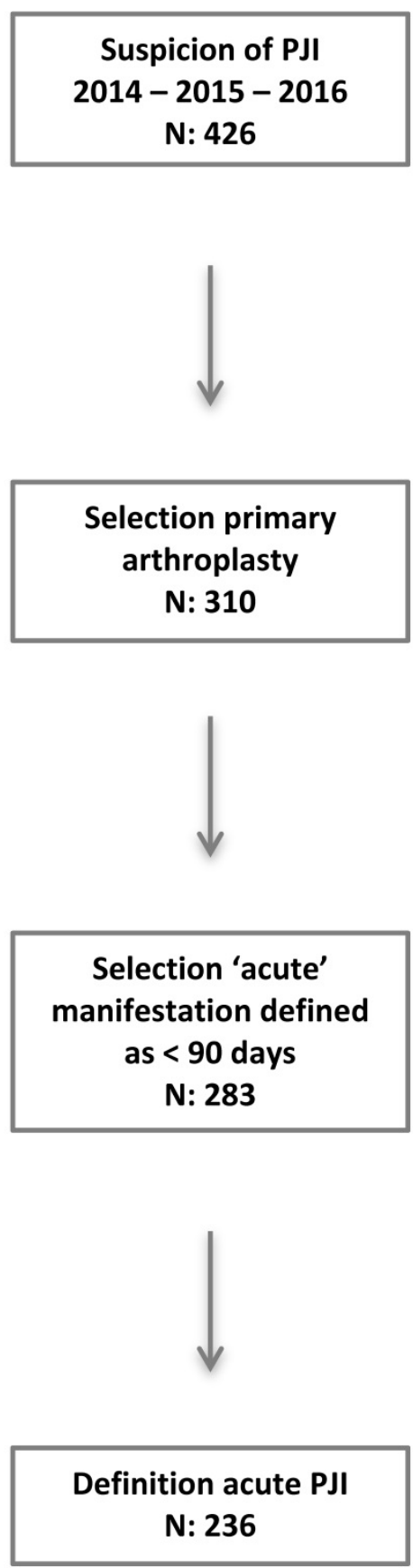

Figure 2. Flow chart patient inclusion

\section{Patient characteristics}

Patient demographics of acute PJI and prosthetic characteristics are presented in Table 1.

PJI occurred in $148(63 \%)$ cases of primary hip arthroplasty and in $88(37 \%)$ cases of primary knee arthroplasty. Patients' mean age at time of infection 
was 68 years (SD 11.5 years), 147 cases (62\%) were male and 89 cases (38\%) were female.

Table 1. Patient demographics, baseline clinical characteristics and prosthetic characteristics.

\begin{tabular}{lll}
\hline Variables & $\mathrm{N}$ & $(\%)$ \\
\hline Joints & & \\
Hip arthroplasty & 148 & $(63 \%)$ \\
Knee arthroplasty & 88 & $(37 \%)$ \\
Sex & & \\
$\quad$ Male & 147 & $(62 \%)$ \\
Female & 89 & $(38 \%)$ \\
Age (years) & 68 & $( \pm 11.5)$ \\
Body mass index $\left(\mathrm{kg} / \mathrm{m}^{2}\right)^{*}$ & 29.9 & $( \pm 5.2)$ \\
Normal [18.5-24.9] & 35 & $(15 \%)$ \\
Overweight [25-29.9] & 99 & $(42 \%)$ \\
Obesity [30-34.9] & 66 & $(28 \%)$ \\
Morbid obesity [ $\geq 35]$ & 36 & $(15 \%)$ \\
Risk factors & & \\
Diabetes mellitus & 15 & $(6 \%)$ \\
Malignancy & 15 & $(6 \%)$ \\
Others (e.g. cardiac history) & 55 & $(23 \%)$ \\
Rheumatoid arthritis & 14 & $(6 \%)$ \\
Smoking & 30 & $(13 \%)$ \\
\hline
\end{tabular}

*presented as mean $( \pm \mathrm{SD})$

\section{Prosthetic Joint Infection characteristics}

The median interval between index procedure and diagnosis of infection was 20 days. On admission, $73 \%$ of the PJI patients had persistent wound leakage and 50\% erythema at the affected joint. Of all PJI patients $49 \%$ had 2 or more clinical features at presentation. The predominant pathogens cultured from intraoperative samples during the first debridement were staphylococcus aureus $(\mathrm{N}=107,45 \%)$, coagulase negative staphylococcus $(\mathrm{N}=99,42 \%)$, streptococcus sp. $(\mathrm{N}=32,14 \%)$ and corynebacterium sp. $(\mathrm{N}=30,13 \%)$. Table 2.

Table 2. Predominant organisms cultured from intraoperative samples during the first debridement.

\begin{tabular}{lll}
\hline Organism & $\mathrm{N}$ & $(\mathrm{N} \%)$ \\
\hline Staphylococcus aureus & 107 & $(45 \%)$ \\
Coagulase negative staphylococcus & 99 & $(42 \%)$ \\
Corynebacterium species & 30 & $(13 \%)$ \\
Streptococcus species & 32 & $(14 \%)$ \\
Haemolyticus group A & 2 & $(1 \%)$ \\
Haemolyticus Group B & 3 & $(1 \%)$ \\
Haemolyticus Group C & 2 & $(1 \%)$ \\
Haemolyticus Group G & 8 & $(3 \%)$ \\
Enterococcus species & 33 & $(14 \%)$ \\
\hline
\end{tabular}

\section{Adherence to the protocol}

Following the DAIR treatment, at the first debridement replacement of exchangeable parts significantly increased $(\mathrm{p}<0.01)$ from $24 / 53$ cases (45\%) in 2014, to $42 / 79$ cases (53\%) in 2015 and $73 / 104$ cases $(70 \%)$ in 2016 . Over the years in less cases more than two times a debridement was performed $(p<0.01)$ and an increase in the number of cultures obtained $(\geq 6)$ from the affected prosthetic joint $(\mathrm{p}<0.001)$ Table 3.

Table 3. Adherence to the regional protocol.

\begin{tabular}{|c|c|c|c|c|c|c|c|c|}
\hline & \multicolumn{2}{|c|}{2014} & \multicolumn{2}{|c|}{2015} & \multicolumn{2}{|c|}{2016} & \multicolumn{2}{|c|}{ Total } \\
\hline & $\mathbf{N}$ & $(\%)$ & $\mathbf{N}$ & $(\%)$ & $\mathbf{N}$ & $(\%)$ & $\mathbf{N}$ & $(\%)$ \\
\hline \multicolumn{9}{|c|}{ No. of debridements } \\
\hline 1 & 30 & $(57 \%)$ & 57 & $(72 \%)$ & 63 & $(60 \%)$ & 150 & $(64 \%)$ \\
\hline 2 & 13 & $(24 \%)$ & 15 & $(19 \%)$ & 38 & $(37 \%)$ & 66 & $(28 \%)$ \\
\hline$\geq 3^{* *}$ & 10 & $(19 \%)$ & 7 & $(9 \%)$ & 3 & $(3 \%)$ & 20 & $(8 \%)$ \\
\hline \multicolumn{9}{|c|}{$\begin{array}{l}{ }^{*} \text { Exchange of } \\
\text { components }\end{array}$} \\
\hline Yes $^{* *}$ & 24 & $(45 \%)$ & 42 & $(53 \%)$ & 73 & $(70 \%)$ & 139 & $(59 \%)$ \\
\hline \multicolumn{9}{|c|}{ No. of cultures } \\
\hline$\geq 6^{* *}$ & 22 & $(42 \%)$ & 58 & $(73 \%)$ & 73 & $(70 \%)$ & 153 & $(65 \%)$ \\
\hline
\end{tabular}

\section{Outcome}

Outcome following DAIR is detailed in Table 4. After 3 months follow-up, prosthesis retention was achieved in $215(91 \%)$ cases and after 12 months follow-up in $205(87 \%)$ cases. This latter percentage remained stable over the years $(p>0.05)$. At the 12 months follow-up $24(10 \%)$ of the patients required some form of surgery post-DAIR, for example a Girdlestone situation, amputation or one/two stage revision. Of our patients with PJI, 3\% (N=7) died within one year after the primary surgery.

Table 4. Outcome following DAIR and complications.

\begin{tabular}{lllll}
\hline & 3 months & $\mathbf{( \% )}$ & 12 months & $\mathbf{( \% )}$ \\
\hline Prosthesis retention & 215 & $(91 \%)$ & 205 & $(87 \%)$ \\
Reoperation & 17 & $(7 \%)$ & 24 & $(10 \%)$ \\
One-stage revision & 6 & $(2.5 \%)$ & 4 & $(1.7 \%)$ \\
Two-stage revision & 3 & $(1.3 \%)$ & 14 & $(5.9 \%)$ \\
Girdlestone & 4 & $(1.7 \%)$ & 4 & $(1.7 \%)$ \\
Amputation & 1 & $(0.4 \%)$ & 1 & $(0.4 \%)$ \\
Unknown & 3 & $(1.3 \%)$ & 0 & $(0 \%)$ \\
Mortality & 4 & $(2 \%)$ & 7 & $(3 \%)$ \\
\hline
\end{tabular}

* In three cases the type of reoperation at three months was not defined.

\section{Discussion}

With this unique collaboration of nine hospitals in the South-East Netherlands we created a first regional registry, based on one combined treatment protocol for acute PJI of THA and TKA. We evaluated adherence to the treatment protocol. Moreover, this is the first study on diagnosis and treatment of PJI, which includes a large number of cases from different hospitals in the Netherlands using such a well-defined protocol.

The PJI incidence within the regional cohort was $1.12 \%$, which is comparable to the range of $0.5-2 \%$ in literature. $[2,3,20]$ The risk factors that are associated with developing PJI following total joint arthroplasty include male sex, age, higher body mass index (BMI), 
and the presence of comorbidities such as diabetes, cardiac history. These factors are also associated with a higher mortality risk, suggesting there would be an association between PJI and mortality. [21] Moreover, the mortality rate was $3 \%$ within one year after total joint arthroplasty infection, in line with literature $(3-4 \%)$. [22,23] For comparison, the relative five-year survival rate for the most common cancers are $99 \%$ for prostate cancer, $89 \%$ for breast cancer, $64 \%$ for colorectal cancer $16 \%$ for lung and bronchial cancer and $91 \%$ for melanoma. [24] The high mortality rates related to PJI support the notion of a devastating complication and should be part of counseling of the patients $[21,23]$.

Several guidelines have been developed to aid the challenging of diagnosis and treatment of PJIs. [e.g. 2,11] The regional protocol was based on these evidence-based statements in order to standardize PJI management. In line with our results, Armstrong et al. (2018) recently demonstrated that adherence to specific aspects of a PJI guideline varies considerably. They suggested that lack of awareness or not recognizing the importance of these aspects might be underlying issues for a not optimal protocol adherence. [12] The implementation of our regional treatment protocol for PJI involved regular discussion between the participating hospitals to get everybody on board, to clarify used definitions and to present analyses of the registered data. Over the course of the collaboration we found an increase in adherence to key-components of the treatment protocol. The better adherence was not yet reflected in better outcome.

\section{Limitations}

The diagnosis of PJI is complex; multiple diagnostic tools are used in the attempt to correctly diagnose PJI. [20] Nowadays, the MSIS criteria are generally accepted worldwide and its use in research allowed for consistency in definition between studies. For the current analyses we used one of the major diagnostic criteria of MSIS; at least two phenotypically identical pathogens isolated from at least two separate tissues to identify true PJI cases. $[2,4,18]$ In recent years, also numerous markers have been evaluated and described as minor criteria for PJI. However, as Parvizi et al. (2018) mentioned, while major criteria for infection are identical between the different definitions, the minor criteria differ and are less agreed upon. Publications in recent years have shown different weights (sensitivity and specificity) for the various tests (minor criteria) used. [18] Furthermore, PJI might be present if fewer criteria are met and even if all perioperative obtained cultures were negative [10]. Unfortunately, as not all minor criteria were integrated in the online database, these data were not available to take into account to identify true PJI. In the current analyses, the definition of PJI was created using the most objective unbiased criteria that identified the strongest major diagnostic criteria. As a result there is no doubt about the accuracy of the PJI diagnosis in the identified cases.

Furthermore, the multicenter design of the study with incorporation of data from multiple centers in the regional PJI database may be caused variation in data interpretation. During the regional meetings we discussed this and reached consensus to minimize missing data. Such comparative data sharing improves the quality of care of PJI. The great variability of the way surgeons diagnose PJI, e.g. the interpretation of subjective assessments of tissue inflammation, is a major reason for underestimating the prosthetic joint infection. [17,18] Although the total number of primary THAs and TKAs performed in the participating hospitals only increased minimally, we noticed an increase in PJIs in our cohort over the three years evaluated. We believe this is rather a sign of better adherence to the protocol and better registration instead of a real increase of PJIs.

\section{Conclusion}

Results of the regional cohort are in line with the available literature. Regional collaboration and regular feedback on registered data resulted in better adherence to the combined treatment protocol. Despite our attempts to improve PJI care, PJI remains a serious complication of THA and TKA with a significant mortality rate and great impact on the patients and health care. Since it is still not possible to determine a reliable incidence of PJI from the (Dutch) arthroplasty register, a specified PJI register is necessary to evaluate incidences and follow-up results. Such a register will enhance the collaboration between hospitals where experience and treatment results are routinely evaluated and discussed, to improve the quality of care around acute PJI of hip and knee arthroplasty.

\section{Acknowledgments}

The authors would like to thank scientific board of the Regional Prosthetic Joint Infection working group for their valuable input on the manuscript.

\section{Regional Prosthetic Joint Infection working group}

The Regional Prosthetic Joint Infection working group includes: W.H.C. Rijnen, J.H.M. Goossen, J.G.E. Hendriks, R. Roerdink, T. Weernink, W. van der Weegen, Y. Pronk, A.M.J. Burgers, S. de Boer. 


\section{Competing Interests}

The authors have declared that no competing interest exists.

\section{References}

1. Zimmerli W, Trampuz A, Ochsner PE. Prosthetic-Joint Infections. N Engl J Med. 2004; 351: 1645-1654. doi:10.1056/NEJMra040181

2. Osmon DR, Berbari EF, Berendt AR, et al. Executive summary: Diagnosis and management of prosthetic joint infection: Clinical practice guidelines by the Infectious Diseases Society of America. Clin Infect Dis. 2013; 56:1-10. doi:10.1093/cid/cis966

3. de Vries L, van der Weegen W, Neve W, Das H, Ridwan B, Steens J. The Effectiveness of Debridement, Antibiotics and Irrigation for Periprosthetic Joint Infections after Primary Hip and Knee Arthroplasty. A 15 Years Retrospective Study in Two Community Hospitals in the Netherlands. J Bone Jt Infect. 2016; 1: 20-24. doi:10.7150/jbji.14075

4. Kuiper JW. Treatment of acute periprosthetic infections with prosthesis retention: Review of current concepts. World J Orthop. 2014; 5: 667-676. doi:10.5312/wjo.v5.i5.667

5. Pulido L, Ghanem E, Joshi A, Purtill JJ, Parvizi J. Periprosthetic joint infection: The incidence, timing, and predisposing factors. Clin Orthop Relat Res. 2008; 466: 1710-1715. doi:10.1007/s11999-008-0209-4

6. Grammatopoulos G, Kendrick B, McNally M, et al. Outcome Following Debridement, Antibiotics, and Implant Retention in Hip Periprosthetic Joint Infection-An 18-Year Experience. J Arthroplasty. 2017; 32: 2248-2255. doi:10.1016/j.arth.2017.02.066

7. Trampuz A, Widmer AF. Infections associated with orthopedic implants. Curr Opin Infect Dis. 2006; 19: 349-356. doi:10.1097/01.qco.0000235161.85925.e8

8. Kuiper JWP, Vos SJ, Saouti R, et al. Prosthetic joint-associated infections treated with DAIR (debridement, antibiotics, irrigation, and retention). Acta Orthop. 2013; 84: 380-386. doi:10.3109/17453674.2013.823589

9. Urish KL, Bullock AG, Kreger AM, et al. A Multicenter Study of Irrigation and Debridement in Total Knee Arthroplasty Periprosthetic Joint Infection: Treatment Failure Is High. J Arthroplasty. 2018; 33: 1154-1159. doi:10.1016/j.arth.2017.11.029

10. Parikh MS, Antony S. A comprehensive review of the diagnosis and management of prosthetic joint infections in the absence of positive cultures. J Infect Public Health. 2016; 9: 545-556. doi:10.1016/j.jiph.2015.12.001

11. Parvizi J, Zmistowski B, Berbari EF, et al. New definition for periprosthetic joint infection: from the Workgroup of the Musculoskeletal Infection Society. Clin Orthop Relat Res. 2011; 469: 2992-2994. doi:10.1007/s11999-011-2102-9

12. Armstrong MD, Carli A V., Abdelbary H, Poitras S, Lapner P, Beaulé PE. Tertiary care centre adherence to unified guidelines for management of periprosthetic joint infections: A gap analysis. Can J Surg. 2018; 61: 34-41. doi:10.1503/cjs.008617

13. Veltman ES, Moojen DJF, Nelissen RG, Poolman RW. Antibiotic Prophylaxis and DAIR Treatment in Primary Total Hip and Knee Arthroplasty, A National Survey in The Netherlands. J Bone Jt Infect. 2018; 3: 5-9. doi:10.7150/jbji.20259

14. Wagenaar F-C, Löwik CAM, Stevens M, et al. Managing persistent wound leakage after total knee and hip arthroplasty. Results of a nationwide survey among Dutch orthopaedic surgeons. J Bone Jt Infect. 2017; 2: 202-207. doi: $10.7150 /$ jbji.22327

15. Viktor Lindgren I, Gordon M, Wretenberg P, Kärrholm J, Garellick G. Validation of reoperations due to infection in the Swedish Hip Arthroplasty Register. BMC Musculoskelet Disord. 2014; 15: 384. doi:10.1186/14712474-15-384

16. Witso E. The rate of prosthetic joint infection is underestimated in the arthroplasty registers. Acta Orthop. 2015; 86: 277-278. doi:10.3109/ 17453674.2015.1042320

17. Gundtoft $\mathrm{PH}$ Overgaard $\mathrm{S}$, Schonheyder $\mathrm{HC}$, Moller JK, Kjærsgaard-Andersen P, Pedersen AB. The "true" incidence of surgically treated deep prosthetic joint infection after 32,896 primary total hip arthroplasties. Acta Orthop. 2015; 86: 326-334. Doi:10.3109/ 17453674.2015.1011983

18. Parvizi J, Tan TL, Goswami K, et al. The 2018 Definition of Periprosthetic Hip and Knee Infection: An Evidence-Based and Validated Criteria. Journal of Arthroplasty. 2018; 33: 1309-1314.

19. LROI (2017, June). Number of primary total arthroplasties registered in the LROI in the Netherland 2010-2016. Retrieved from: http://www.lroirapportage.nl/hip-numbers-procedures-2010-2017.

20. Mühlhofer HML, Pohlig F, Kanz KG, et al. Prosthetic joint infection development of an evidence-based diagnostic algorithm. Eur J Med Res. 2017; 22:8. doi:10.1186/s40001-017-0245-1

21. Zmistowski B, Karam JA, Durinka JB, Casper DS, Parvizi J. Periprosthetic joint infection increases the risk of one-year mortality. J Bone Jt Surg Am. 2013; 95: 2177-2184. doi:10.2106/JBJS.L.00789

22. Natsuhara KM, Shelton TJ, Meehan JP, Lum ZC. Mortality During Total Hip Periprosthetic Joint Infection. J Arthroplasty. 2018; [Epub ahead of print]. doi:10.1016/j.arth.2018.12.024
23. Lum ZC, Natsuhara KM, Shelton TJ, Giordani M, Pereira GC, Meehan JP. Mortality During Total Knee Periprosthetic Joint Infection. J Arthroplasty. 2018; 33: 3783-3788. doi:10.1016/j.arth.2018.08.021

24. American Cancer Society, Surveillance Research (2012). Cancer Facts \& Figures 2012 Supplemental Data. Retrieved from: https://www.cancer.org/ research/cancer-facts-statistics/all-cancer-facts-figures/cancer-facts-figures-2 012.html. 